\title{
Surgical Intervention to Rescue Hirschsprung Disease in a Rat Model
}

\author{
Lincon A Stamp, ${ }^{1 *}$ Florian Obermayr, ${ }^{1,2}$ Louise Pontell, ${ }^{1}$ Heather M Young, ${ }^{1}$ Dan Xie, ${ }^{3}$ David H Croaker, ${ }^{4}$ Zan-Min Song, ${ }^{3}$ and \\ John B Furness ${ }^{1}$
}

${ }^{1}$ Department of Anatomy and Neuroscience, University of Melbourne, Parkville, VIC, Australia; ${ }^{2}$ Department of Pediatric Surgery and Pediatric Urology, University Children's Hospital Tuebingen, Tuebingen, Germany; ${ }^{3}$ Eccles Institute of Neuroscience, John Curtin School of Medical Research and Medical School, Australian National University, Canberra, ACT, Australia; and ${ }^{4}$ Department of Pediatrics and Child Health, Canberra Hospital, Canberra, Australia

\section{Background/Aims}

Rats with a spontaneous null mutation in endothelin receptor type B or Ednrb (s//sl; spotting lethal) lack enteric neurons in the distal bowel and usually die within the first week after birth. This early postnatal lethality limits their use for examining the potential of cell therapy to treat Hirschsprung disease, and for studies of the influence of EDNRB on the mature CNS and vascular systems.

\section{Methods}

We have developed a surgical intervention to prolong the life of the spotting lethal s/s/ rat, in which we perform a colostomy on postnatal (P) day 4-6 rats to avoid the fatal obstruction caused by the lack of colonic enteric neurons.

\section{Results}

The stomas remained patent and functional and the rats matured normally following surgery. Weight gains were comparable between control and Hirschsprung phenotype (s//s/) rats, which were followed until 4 weeks after surgery (5 weeks old). We confirmed the absence of enteric neurons in the distal colon of rats whose lives were saved by the surgical intervention.

\section{Conclusions}

This study provides a novel approach for studying EDNRB signalling in multiple organ systems in mature rats, including an animal model to study the efficacy of cell therapy to treat Hirschsprung disease.

\section{(J Neurogastroenterol Motil 2015;21:552-559)}

\section{Key Words}

Colostomy; Endothelin signalling; Enteric nervous system; Hirschsprung disease

Received: May 11, 2015 Revised: June 17, 2015 Accepted: June 30, 2015

(c) This is an Open Access article distributed under the terms of the Creative Commons Attribution Non-Commercial License (http://creativecommons. org/licenses/by-nc/4.0) which permits unrestricted non-commercial use, distribution, and reproduction in any medium, provided the original work is properly cited.

\section{*Correspondence: Lincon A Stamp, PhD}

Department of Anatomy and Neuroscience, Medical School Building, University of Melbourne, Grattan Street, Parkville 3010, Australia

Tel: +61-3-8344-5783, Fax: +61-3-9035-8837, E-mail: Istamp@unimelb.edu.au

Financial support: This work was funded by NHMRC Project grants (No.1019931 and 1079234) to Heather M Young, Lincon A Stamp, and John B Furness. We also acknowledge the assistance of a donation from The Bootes Foundation to Zan-Min Song and David $\mathrm{H}$ Croaker. Conflicts of interest: None.

Author contributions: Lincon A Stamp, John B Furness, Heather M Young, and David H Croaker conceived and designed the experiments; Lincon A Stamp, Florian Obermayr, Louise Pontell, John B Furness, Zan-Min Song, and Dan Xie performed the experiments; Lincon A Stamp, John B Furness, and Heather M Young analysed the data; and Lincon A Stamp, John B Furness, and Heather M Young wrote the paper. All authors contributed to the final manuscript.

ORCID: Heather M Young, http://orcid.org/0000-0002-0457-8710. 


\section{Introduction}

The enteric nervous system (ENS) is a complex network of neurons and glia in the wall of the gastrointestinal tract that plays important roles in the control of motility, blood flow and secretion. ${ }^{1,2}$ The ENS is mostly derived from neural crest-derived cells that emigrate from the caudal hindbrain, enter the foregut and then migrate caudally to colonise the entire gastrointestinal tract. $^{3-5}$ Failure of the enteric neural crest cells (ENCCs) to fully colonise the bowel results in Hirschsprung disease (HSCR), or congenital aganglionosis, in which intrinsic neurons are absent from the distal bowel, resulting in an absence of propulsive gut motility patterns and intractable constipation. ${ }^{6-11}$ Standard, current treatment for most HSCR patients involves a one-stage surgical resection of the defective portion of bowel and reanastomosis of the functional bowel to the anal canal using transanal and laparoscopic approaches. ${ }^{12,13}$ Although patients suffering from recto-sigmoid length HSCR can generally be treated without enterostomy, those with long-segment disease, intractable ileus, enterocolitis, extremely enlarged megacolon, and/or malnutrition ${ }^{14}$ require a first stage reversible enterostomy to allow for improved health of the child prior to a second surgery to remove the aganglionic colon and to join the functioning ganglionic bowel to the anal canal. ${ }^{15}$ Because many patients suffer chronic complications, including constipation and fecal soiling, following surgery, ${ }^{16}$ there has been much interest in exploring the potential of cell therapy to replace the missing enteric neurons and restore gut motility in animal models of HSCR. ${ }^{17-21}$

Rats with a spontaneous null mutation in endothelin receptor type B, Ednrb (sl/sl; spotting lethal) lack enteric neurons in the distal bowel and hence display a HSCR-like phenotype. ${ }^{22,23}$ Because of their size and the ability to examine colonic motility in vivo, ${ }^{24,25}$ rats are an attractive animal model to examine the potential of cell therapy to treat HSCR. However, Ednrb null mutant rats usually die within the first week after birth due to intestinal obstruction and associated enterocolitis. ${ }^{22,23}$ The perinatal lethality of Ednrb null mutant rats restricts their use for studies of the effect of lack of EDNRB on mature CNS and vascular systems as well as for examining cell therapy for HSCR.

Here we describe a surgical intervention on P4-P6 rats lacking neurons from most of the colon that prolongs their life by preventing the fatal obstruction. This procedure will be useful for studying the role of EDNRB signalling in mature rats and for studies examining the potential of cell therapy for Hirschsprung disease.

\section{Materials and Methods}

\section{Animals}

Data were obtained from the Wistar-Imamichi, congenital aganglionosis rat (AR) strain, which lack a functional EDNRB. ${ }^{22,26}$ Litters of $+/+,+/ \mathrm{sl}$, and $\mathrm{sl} / \mathrm{sl}$ rats were generated by mating heterozygous $(+/ \mathrm{sl})$ with Wistar rats for 2 generations, then intercrossing $+/$ sl rats, from a colony at the Canberra Hospital animal facility. Pups were obtained at postnatal (P) day 4-6. Due to backcrossing with Wistar rats, coat colour was not a reliable indicator of aganglionosis. Therefore, following the experimental period, all rats were assessed for the presence of intrinsic enteric neurons in the distal bowel by immunohistochemistry (see below). Genotyping using a PCR-based assay was not performed because this assay does not provide information about the length of the aganglionic region. All studies were approved by the Anatomy and Neuroscience, Pathology, Pharmacology, and Physiology Animal Ethics Committee of the University of Melbourne (ethics ID 1112288).

\section{Colostomy Surgery}

A total of 21 rats at the age of P4-6 were anaesthetised with $3 \%$ isoflurane vapourised in oxygen, following induction with $5 \%$ isoflurane. An incision was made to the left of the midline (Fig. 1). The cecum was internally reflected to expose the proximal colon (Fig. 1). The proximal colon was immobilised by passing 5-0 braided, polyglycolic acid suture (BBraun, Bella Vista, NSW, Australia) through the abdominal wall and under the bowel to close the muscle under the proximal colon. The colon was anchored to the abdominal wall, anteriorly and posteriorly, using 8-0 braided, polyglycolic acid suture (BBraun). The bowel was then completely severed, the fecal contents thoroughly removed from the site and the skin between the future rostral and caudal stomas was closed using 8-0 braided, polyglycolic acid suture. Without removal of any segment of colon, the anterior stoma (proximal colon leading to the anus) and posterior stoma (proximal colon leading to the cecum and small intestine) were each fixed to the skin in 4 locations around the stoma circumference using the 8-0 suture. The skin was then completely closed around the stomas with the same suture, being careful not to constrict the opening of the stoma. Woundgard (Virbac, vet-n-pet Direct, Jimboomba, Qld, Australia), a bitter tasting antiseptic cream that prevents the mother licking the wound, was applied twice daily for the first 3 

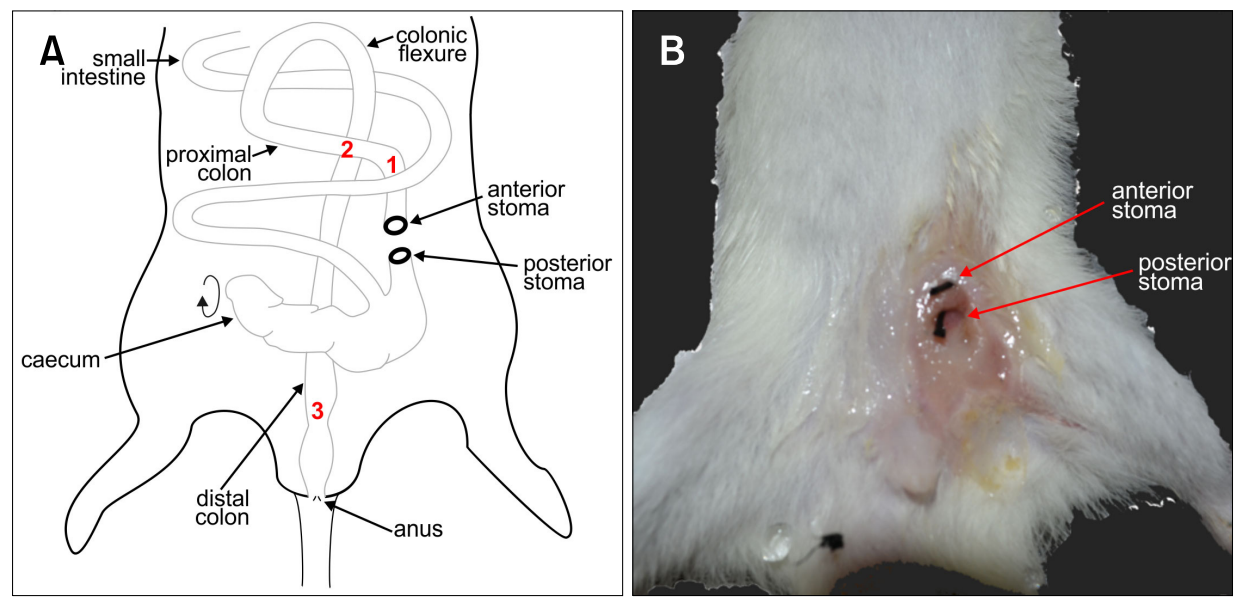

Figure 1. Schematic of rat colostomy and macroscopic external image of the stomas after 4 weeks. (A) Schematic of the anatomy of the rat intestine following colostomy and formation the anterior and posterior stomas, with the cecum rotated in order to locate the proximal colon and bring it to the ventral surface to form the stomas. Red numbers refer to regions processed for immunohistochemistry and shown in Fig. 3. (B) Macroscopic image of the stoma site 4 weeks after colostomy. The 2-0 suture (black threads) has been inserted into the anterior and posterior stomas, to show their positions and to demonstrate that they remained patent.

days. After regaining consciousness, the pups were wiped thoroughly with the mother's bedding and fecal pellets before being returned to the mother. Animals were then monitored and weighed twice daily for 28 days.

\section{Histology and Immunofluorescence Microscopy}

Rats were killed by $\mathrm{CO}_{2}$ asphyxiation 28 days after colostomy surgery. The stoma site including the surrounding skin, a region of proximal colon immediately adjacent to the stoma, and a region of distal colon were resected and fixed in $2 \%$ formaldehyde plus $0.2 \%$ picric acid in $0.1 \mathrm{M}$ sodium phosphate buffer, $\mathrm{pH} 7.2$, overnight at $4^{\circ} \mathrm{C}$. Each sample was placed in a histology cassette, dehydrated through graded ethanol to histolene and embedded in paraffin. Sections were cut transversely at a thickness of $5 \mu \mathrm{m}$ and processed for standard hematoxylin and eosin (H\&E) staining. Wholemount preparations of regions of proximal and distal colon were also made by opening the colon along the mesenteric border, pinning, stretching on Sylgard-coated dishes, and fixing in $4 \%$ paraformaldehye in $0.1 \mathrm{M}$ phosphate buffer overnight. The tissue was then washed, the mucosa removed and the external muscle layers were exposed to $0.1 \%$ Triton X-100 for 30 minutes. The tissue was then incubated with human anti-Hu C/D (1:5000; gift of Vanda Lennon, Mayo Clinic, Rochester, Minnesota, USA) and mouse anti-Tuj1 (1:2000; Covance, Princeton, New Jersey, USA) primary antibodies, followed by donkey anti-human Alexa Fluor 594 (1:500; Life Technologies, Mulgrave, Victoria,
Australia) and donkey anti-mouse Alexa Fluor 488 (1:200; Life Technologies) secondary antibodies, all at room temperature.

Images of $\mathrm{H} \& \mathrm{E}$ stained sections were taken using a Mirax digital scanning microscope (Zeiss, Sydney, Australia).

\section{Results}

To by-pass the fatal colonic obstruction caused by the Ednrb null mutation in $s l / s l$ rats, a colostomy was performed on P4-6 rats. A stoma was formed by severing the proximal colon and suturing both cut ends of the colon to the abdominal wall. In order to reveal the proximal colon and bring it to the ventral abdominal surface, the cecum was reflected caudally (Fig. 1). This means that the stoma towards the rat's tail (posterior in the rat) was distal to the cecum and the digesta that was conveyed from the small intestine and cecum was released from this stoma. The anterior stoma led to the anus (Fig. 1A and 1B). Both anterior and posterior stomas remained patent (Fig. 1B).

Preliminary studies revealed some early mortality due to the mother licking the wounds. This was prevented by applying Woundgard, an antiseptic, bitter-tasting cream to the wound. Following colostomy, the rats were weighed twice daily and the stomas examined. Any fecal plugs in the stomas were removed using cotton tips. Ten of 21 pups died due to rejection and cannibalism by the mother within 72 hours of surgery. A total of $4 \mathrm{sl} / \mathrm{sl}$ and 7 control rats $(+/+$ and $+/ s l)$ in this series were allowed to survive for 4 weeks following colostomies (note that rats were 
confirmed to be $s l / s l$ when immunohistochemistry was performed on the colon after being killed 4 weeks after surgery). Four $s l / s l$ and 9 control rats also survived to 4 weeks after surgery in another series (see Discussion). Following surgery, rats grew and matured normally and they ate solid food from 3 weeks old. There was a slight but significant difference in average weight between null mutant and control rats at 7 and 14 days following colostomy (Fig. 2), but by 21 and 28 days after surgery, there was no significant difference in the weights of mutant and control animals (Fig. 2). However, sl/sl and control rats that had undergone colostomies weighed around 30-40\% less than un-operated siblings (un-operated siblings $[\mathrm{n}=5]$, weighed only at 28 days). There was occasional mild irritation to the skin of the hindlimb on the stoma side of rats that had undergone colostomies. This is likely due to prolonged exposure of the hindlimb to the fecal contents expelled from the stoma.

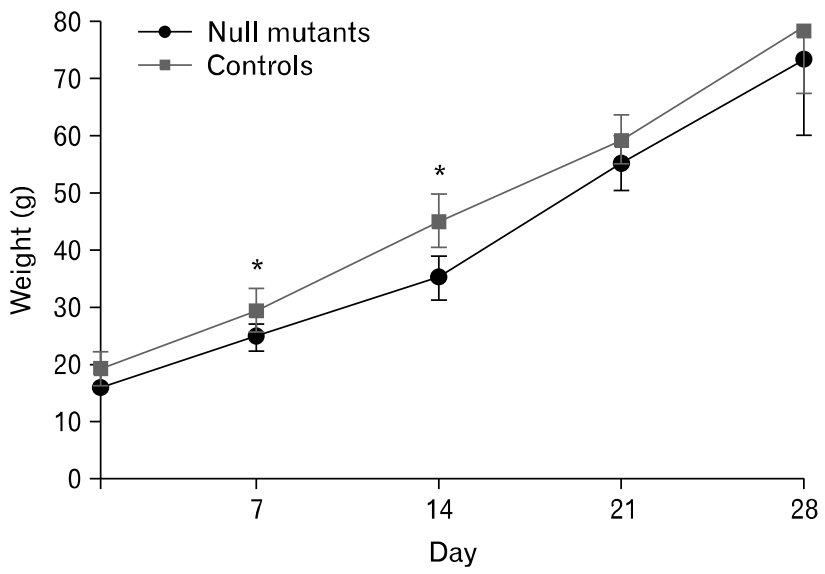

Figure 2. Weight gain chart of $s l / s l$ mutants and control rats after stoma surgery. Following surgery, mutant $(\mathrm{n}=4)$ and control $(\mathrm{n}=7)$ rats were monitored and weighed twice daily for 28 days. Data represents the mean weight $\pm \mathrm{SD} .{ }^{*} P<0.05$ : t test.
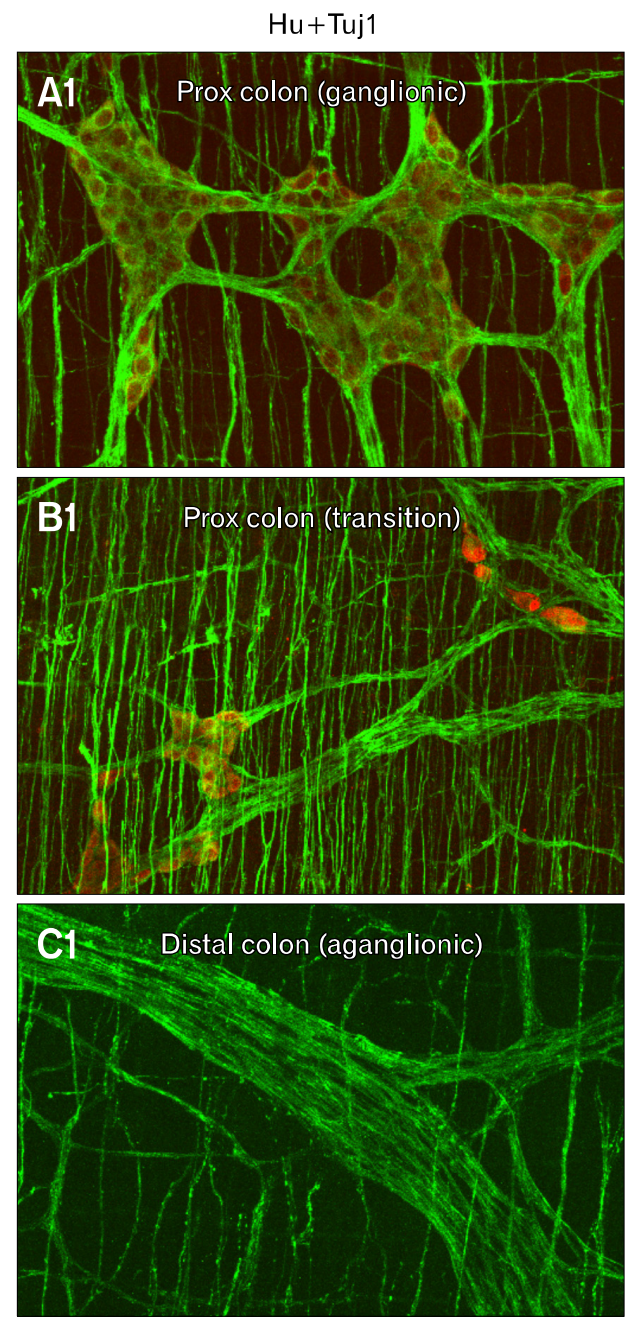

$\mathrm{Hu}$

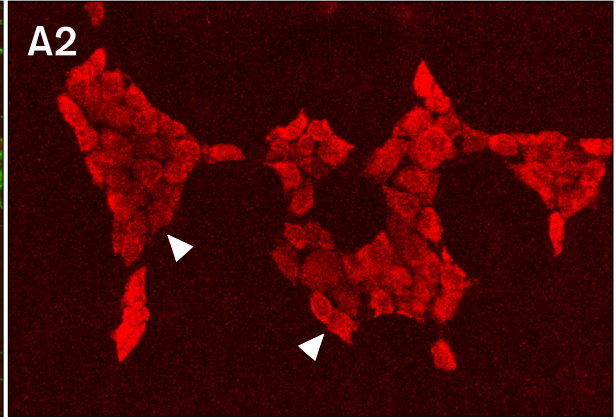

B2

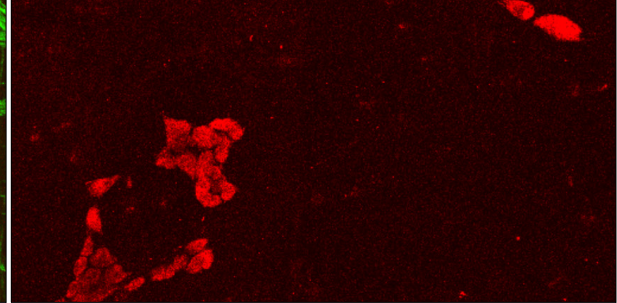

\section{C2}

Vol. 21, No. 4 October, 2015 (552-559)
Figure 3. Immunohistochemical demonstration of aganglionosis in an $s l / s l$ rat. Immunostaining using antibodies to the nerve fiber marker, Tuj1 (green), and the nerve cell body marker, $\mathrm{Hu}$ (red), of wholemount preparations of circular and longitudinal muscle layers from the proximal colon adjacent to the cecum (A1, A2: region "1" in Fig. 1), proximal colon $\sim 1-2 \mathrm{~cm}$ distal to the cecum $(\mathrm{B} 1$, B2: region "2" in Fig. 1) and distal colon of an $s l / s l$ rat (C1, C2: region "3" in Fig. 1). (A1, A2) In the proximal colon adjacent to the cecum (marked 1 on Fig. 1), $\mathrm{Hu}+$ neurons form myenteric ganglia (arrowheads) between the longitudinal and circular muscle layers, and Tuj1 + fibers are present in the ganglia and the circular muscle layer. (B1, B2) Slightly caudal in the proximal colon (marked 2 on Fig. 1) is the transition zone, which is a region of reduced $\mathrm{Hu}+$ neuron density. (C1, C2) In the distal colon (marked 3 on Fig. 1), there are no $\mathrm{Hu}+$ neurons present $(\mathrm{C} 2)$, but there are some thick nerve fiber trunks and there are also fibers in the circular muscle layer, but at lower density than in the ganglionated region and transition zone of the proximal colon. Scale bar: $50 \mu \mathrm{m}$. 
Four weeks following colostomy surgery, rats were killed and examined. Dissection of the stoma region showed good continuity between the bowel and the skin, with no evidence of abscesses or other lesions. Internally, there were no adhesions of the operated bowel to other regions of intestine or to other abdominal organs and no indication of peritonitis. There was no dilation of the proximal colon oral to the stoma, that is, no evidence of a megacolon.

Wholemount preparations of proximal colon and distal colon from each rat were examined by immunofluorescence using antibodies to pan-neuronal markers, $\mathrm{Hu} \mathrm{C} / \mathrm{D}$ (cell bodies), and Tuj1 (cell bodies and intrinsic/extrinsic nerve fibers), to identify mutants. All $4 \mathrm{sl} / \mathrm{sl}$ mutant rats possessed $\mathrm{Hu}+$ neurons forming ganglia in the proximal colon (Fig. 3A1 and 3A2). In the proximal colon distal to the ganglionic region there was a transition zone with a reduced number of $\mathrm{Hu}+$ enteric neurons (Fig. 3B1 and 3B2). In all 4 null mutant rats, $>80 \%$ of the colon lacked $\mathrm{Hu}+$ neurons. In the distal colon, there was a complete absence of $\mathrm{Hu}+$ intrinsic, enteric neurons, but an abundance of Tuj1 nerve fibers, which are presumed to be of extrinsic origin (Fig. $3 \mathrm{C} 1$ and $3 \mathrm{C} 2$ ). This pattern of aganglionosis is indicative of the $s l$ Hirschsprung disease-like phenotype.

Stomas were created in both control and $s l / s l$ mutant rats. The stoma sites, the proximal colon immediately adjacent to the ante-
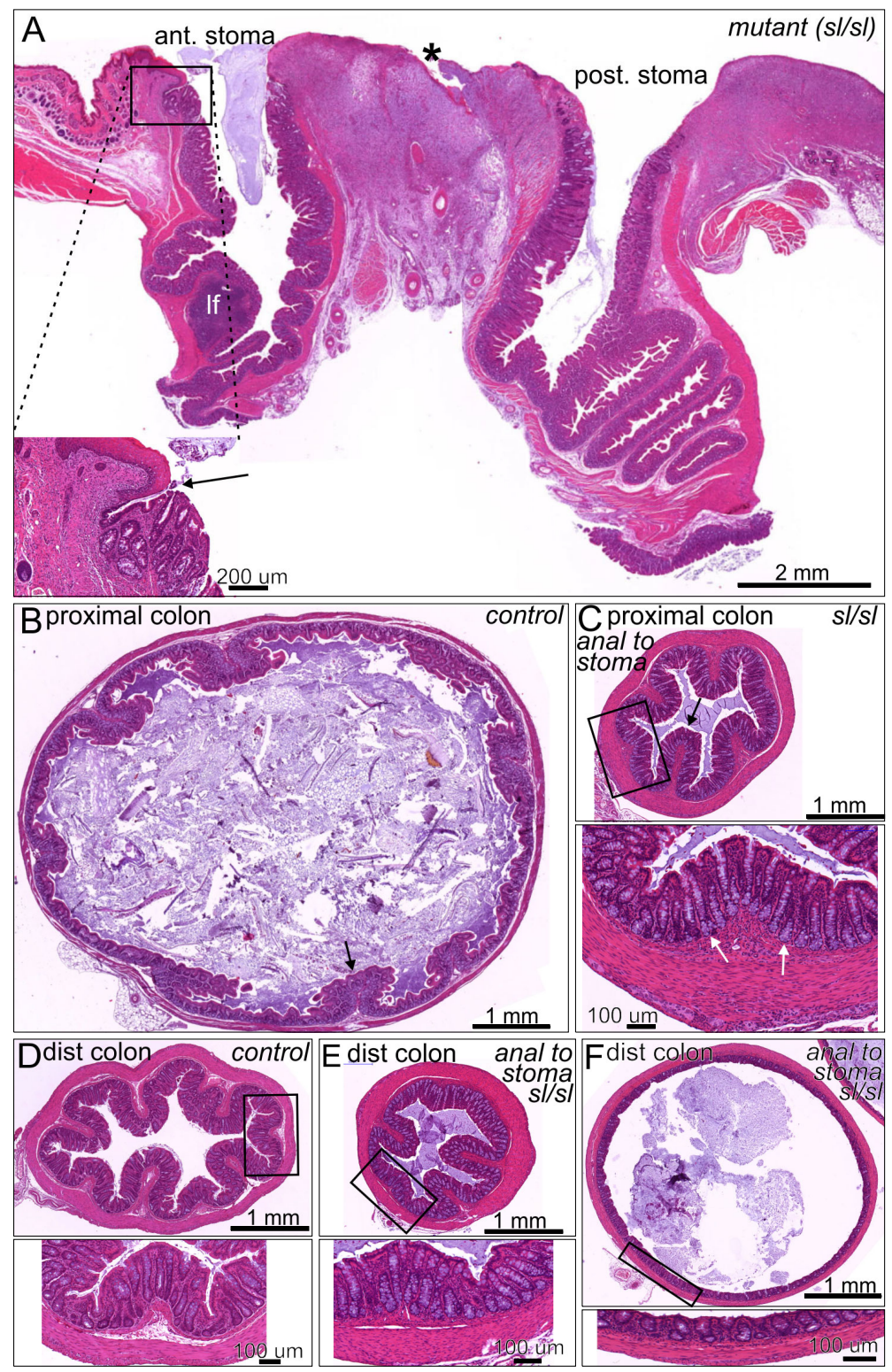

Figure 4. Histology of the stoma region and colon 4 weeks after the creation of a dual colostomy in mutant rats. (A) Section through the 2 stomas from a rat with Hirschsprung disease phenotype. There is a sharp transition between the colonic mucosa and the epithelium of the skin (arrow on inset). Small areas of erosion occurred in the skin (asterisk), but there was no evidence of abnormality of the epithelial lining of the proximal colon oral or anal to the surgery. A lymphatic follicle $(l f)$ with normal appearance is seen oral to the surgical intervention. (B) Transverse section through the proximal colon from an unoperated control rat. The lumen is large and filled with digesta and the muscle is relatively thin compared to the constricted colon anal to the stoma (panel C). Mucosal folds are apparent (example arrowed). (C) Section through the proximal colon anal to the anterior stoma of a $\mathrm{s} / \mathrm{sl}$ rat. The lumen is smaller and the muscle is thickened compared to the usual appearance of the proximal colon. The mucosa has normal appearance with well formed glands containing a normal complement of goblet cells (arrows in inset). (D-F) distal colon from a control rat (D), and constricted $(\mathrm{E})$ and dilated $(\mathrm{F})$ regions anal to the anterior stoma, from $s l / s l$ rats. The wall structure is normal in both examples from the Hirschsprung phenotype rats, although the thicknesses of the layers are variable according to whether there is constriction or dilation. 
rior stoma and the distal colon were sectioned and assessed by standard H\&E histology. Both the anterior and posterior stomas were clearly patent, although the anterior stoma, which no longer receives digesta from more proximal regions, contained a mucous plug (Fig. 4A). The colonic epithelium formed a clean junction with the skin, although there was some erosion evident in the surrounding skin and some low level inflammation of the underlying connective tissue (Fig. 4A), which is likely due to irritation from exposure to the fecal content and licking to clean this site by the mother and operated rat. When compared to a similar region of proximal colon from an un-operated control rat (Fig. 4B), the proximal colon immediately adjacent to the anterior stoma appeared constricted with thickened musculature (Fig. 4C). The appearance of the mucosal lining was normal, with well-formed glands and goblet cells (Fig. 4 C, inset). Histological analyses of the proximal colon did not reveal any inflammation in either $s l / s l$ or control rats. When compared to un-operated control distal colon (Fig. 4D), the distal colons of both $s l / s l$ and control rats following colostomy were variable in morphology. In some regions the distal colon was constricted with thick muscle and deep epithelial folds (Fig. 4E), while in other regions the external muscle was thin and the mucosa was stretched (Fig. 4F). The colon distal to the stoma in all rats following colostomy had large amounts of mucous within the lumen. The mucosa appeared healthy.

\section{Discussion}

Rats lacking functional EDNRB ( $s l / s l$ rats) have a Hirschsprung disease phenotype. They fail to thrive and the majority die in the first week after birth, ${ }^{22}$ with mortality reaching $100 \%$ by 4 weeks. ${ }^{26}$ We have found that the creation of a colostomy of the proximal colon just anal to the cecum prevented the formation of a megacolon and allowed the rats to grow and mature, indistinguishable from rats without aganglionosis, and survive in good health to the time of harvesting at 5 weeks of age, when all un-operated $s l / s l$ rats are usually dead. ${ }^{26}$ Immunohistochemistry confirmed that the rats that were rescued by the surgery lacked enteric ganglia in the bypassed distal colon. Following the surgical procedure, fecal content was expelled from the stoma in the proximal colon, and the fatal obstruction and associated enterocolitis caused by aganglionosis of the colon in $s l / s l$ rats did not occur.

The colon beyond the stoma was no longer exposed to the products of digestion that would normally arrive from more proximal regions. The proximal colon immediately adjacent to the anterior stoma appeared slightly constricted, with thickened muscle layers. Previous studies have demonstrated that an absence of propulsive motility is associated with hypertrophy of the intestinal muscle. ${ }^{27}$ Furthermore, studies of rodents with mutations in endothelin-3 signalling pathways have shown hypertrophy of the intestinal muscle in the aganglionic regions where there is no propulsive motility. ${ }^{28,29}$ The prolonged absence of exposure to digesta and consequent reduction in propulsive motility is likely to account for the thickening and constriction of the proximal colon observed in the current study. Some regions of the distal colon of operated rats were dilated, and we do not know the reason for this dilatation. However, overall, the wall of the distal colon appeared healthy. In future studies, enteric neural progenitors could be transplanted into this region, with the intention of generating functional enteric neurons and restoring function. Enteric neuron precursors survive and differentiate when transplanted into the aganglionic regions of Hirschsprung mice, ${ }^{18,30-32}$ so survival and differentiation can be expected in rats. It will be necessary to conduct experiments over a longer time period with enteric neuron transplants to determine whether functional control of motility can be restored to the previously aganglionic colon, and whether a segment of colon with restored enteric neurons can be rejoined to the more proximal colon and function normally. We are currently in the process of developing methods to label rat enteric neural progenitors so they can be transplanted into the aganglionic region of $s l / s l$ rats that have been rescued by the creation of a stoma. The described surgical intervention could also be used to prolong the lives of rats lacking EDNRB in order to investigate the roles of EDNRB in the brain ${ }^{33,34}$ and in angiogenesis. ${ }^{35}$

Hirschsprung-associated enterocolitis (HAEC) is a major problem in infants with HSCR. Pull-through surgery has been performed on Ednrb ${ }^{-1-}$ mice to remove the aganglionic segment and prolong life, but this procedure did not prevent the development of HAEC. ${ }^{36}$ In the current study, however, histological analyses of the proximal colon did not reveal any inflammation in either $s l / s l$ or control rats.

This is not the first description of colostomy in rodents. A previous study developed a method of performing colostomies and generating blind colorectal pouches in wildtype mice to provide a site for the introduction of carcinogens or implantation of tumour cells for studies of colon carcinogenesis. ${ }^{37}$ Moreover, a pull-through procedure was developed for Ednrb ${ }^{-/-}$mice to study Hirschsprung-associated enterocolitis

The data presented here were from 4 null mutant and 7 control rats surviving at least 4 weeks following colostomy by author L.A.S. at the University of Melbourne. An additional 4 null mu- 
tant and 9 control rats received colostomies by Z.M.S. and D.X. at the Australian National University. Although weights were not regularly monitored or histological analysis performed, the rats survived until 4 weeks of age when they were killed. Nonetheless, further optimisation of the surgical procedure is possible, for example, middle-line stomas could potentially reduce the incidence of irritation to the skin of the hindlimb caused by prolonged exposure to fecal contents.

In conclusion, given their size and the ability to examine colonic motility in vivo, rats are an attractive animal model to use to examine the potential of cell therapy to treat HSCR. Here we have demonstrated a surgical intervention to prolong the life of Ednrb null mutant, HSCR-model rats, which normally die within the first weeks of life. These rescued animals will allow the study of the potential of cell therapy to restore gut motility in aganglionic bowel, as well as examination of the role of endothelin signalling through Ednrb in the adult CNS and vascular systems.

\section{Acknowledgements}

We are grateful for the use of facilities of the Histopathology and Organ Pathology Service of the Australian Phenomics Network.

\section{References}

1. Gershon MD. The Second Brain. New York: Harper Collins 1998.

2. Furness JB. The enteric nervous system and neurogastroenterology. Nat Rev Gastroenterol Hepatol 2012;9:286-294.

3. Yntema CL, Hammond WS. The origin of intrinsic ganglia of trunk viscera from vagal neural crest in the chick embryo. J Comp Neurol 1954;101:515- 541.

4. Le Douarin NM, Teillet MA. The migration of neural crest cells to the wall of the digestive tract in avian embryo. J Embryol Exp Morphol 1973;30:31-48.

5. Barlow AJ, Wallace AS, Thapar N, Burns AJ. Critical numbers of neural crest cells are required in the pathways from the neural tube to the foregut to ensure complete enteric nervous system formation. Development 2008;135:1681-1691.

6. Whitehouse FR, Kernohan JW. Myenteric plexus in congenital megacolon; study of 11 cases. Arch Intern Med 1948;82:75-111.

7. Heanue TA, Pachnis V. Enteric nervous system development and Hirschsprung's disease: advances in genetic and stem cell studies. Nat Rev Neurosci 2007;8:466-479.

8. McKeown SJ, Stamp L, Hao MM, Young HM. Hirschsprung Disease: a developmental disorder of the enteric nervous system. Wiley Interdiscip Rev Dev Biol 2013;2:113-129.

9. Obermayr F, Hotta R, Enomoto H, Young HM. Development and developmental disorders of the enteric nervous system. Nat Rev
Gastroenterol Hepatol 2013;10:43-57.

10. Butler Tjaden NE, Trainor PA. The developmental etiology and pathogenesis of Hirschsprung disease. Transl Res 2013;162:1-15.

11. Lake JI, Heuckeroth RO. Enteric nervous system development: migration, differentiation, and disease. Am J Physiol Gastrointest Liver Physiol 2013;305:G1-G24.

12. Haricharan RN, Georgeson KE. Hirschsprung disease. Semin Pediatr Surg 2008;17:266-275.

13. Langer JC. Hirschsprung disease. Curr Opin Pediatr 2013;25:368374.

14. Demehri FR, Halaweish IF, Coran AG, Teitelbaum DH. Hirschsprung-associated enterocolitis: pathogenesis, treatment and prevention. Pediatr Surg Int 2013;29:873-881.

15. Somme S, Langer JC. Primary versus staged pull-through for the treatment of Hirschsprung disease. Semin Pediatr Surg 2004;13: 249-255.

16. Rintala RJ, Pakarinen MP. Long-term outcomes of Hirschsprung's disease. Semin Pediatr Surg 2012;21:336-343.

17. Hotta R, Natarajan D, Thapar N. Potential of cell therapy to treat pediatric motility disorders. Semin Pediatr Surg 2009;18:263-273.

18. Hotta R, Stamp LA, Foong JP, et al. Transplanted progenitors generate functional enteric neurons in the postnatal colon. J Clin Invest 2013;123:1182-1191.

19. Kulkarni S, Becker L, Pasricha PJ. Stem cell transplantation in neurodegenerative disorders of the gastrointestinal tract: future or fiction? Gut 2012;61:613-621.

20. Schäfer KH, Micci MA, Pasricha PJ. Neural stem cell transplantation in the enteric nervous system: roadmaps and roadblocks. Neurogastroenterol Motil 2009;21:103-112.

21. Burns AJ, Thapar N. Neural stem cell therapies for enteric nervous system disorders. Nat Rev Gastroenterol Hepatol 2014;11:317-328.

22. Gariepy CE, Cass DT, Yanagisawa M. Null mutation of endothelin receptor type $\mathrm{B}$ gene in spotting lethal rats causes aganglionic megacolon and white coat color. Proc Natl Acad Sci USA 1996;93:867872.

23. Kunieda T, Kumagai T, Tsuji T, Ozaki T, Karaki H, Ikadai H. A mutation in endothelin-B receptor gene causes myenteric aganglionosis and coat color spotting in rats. DNA Res 1996;3:101-105.

24. Ferens DM, Chang EC, Bogeski G, Shafton AD, Kitchener PD, Furness JB. Motor patterns and propulsion in the rat intestine in vivo recorded by spatio-temporal maps. Neurogastroenterol Motil 2005;17:714-720.

25. Shimizu Y, Chang EC, Shafton AD, et al. Evidence that stimulation of ghrelin receptors in the spinal cord initiates propulsive activity in the colon of the rat. J Physiol 2006;576(Pt 1):329-338.

26. Ceccherini I, Zhang AL, Matera I, et al. Interstitial deletion of the endothelin-B receptor gene in the spotting lethal (sl) rat. Hum $\mathrm{Mol}$ Genet 1995;4:2089-2096.

27. Gabella G. Hypertrophy of intestinal smooth muscle. Cell Tissue Res 1975;163:199-214.

28. Spencer NJ, Bayguinov P, Hennig GW, et al. Activation of neural circuitry and $\mathrm{Ca}^{2+}$ waves in longitudinal and circular muscle during CMMCs and the consequences of rectal aganglionosis in mice. Am J Physiol Gastrointest Liver Physiol 2007;292:G546-G555.

29. Won KJ, Torihashi S, Mitsui-Saito M, et al. Increased smooth muscle contractility of intestine in the genetic null of the endothelin ETB 
receptor: a rat model for long segment Hirschsprung's disease. Gut 2002;50:355-360.

30. Anitha M, Joseph I, Ding X, et al. Characterization of fetal and postnatal enteric neuronal cell lines with improvement in intestinal neural function. Gastroenterology 2008;134:1424-1435.

31. Kruger GM, Mosher JT, Tsai YH, et al. Temporally distinct requirements for endothelin receptor $\mathrm{B}$ in the generation and migration of gut neural crest stem cells. Neuron 2003;40:917-929.

32. Nishikawa R, Hotta R, Shimojima N, et al. Migration and differentiation of transplanted enteric neural crest-derived cells in murine model of Hirschsprung's disease. Cytotechnology 2015;67:661-670.

33. Shinohara H, Udagawa J, Morishita R, et al. Gi2 signaling enhances proliferation of neural progenitor cells in the developing brain. J Biol Chem 2004;279:41141-41148.
34. Vidovic M, Chen MM, Lu QY, et al. Deficiency in endothelin receptor $\mathrm{B}$ reduces proliferation of neuronal progenitors and increases apoptosis in postnatal rat cerebellum. Cell Mol Neurobiol 2008;28: 1129-1138.

35. Tanaka T, Sho M, Takayama T, et al. Endothelin B receptor expression correlates with tumour angiogenesis and prognosis in oesophageal squamous cell carcinoma. Br J Cancer 2014;110:1027-1033.

36. Zhao L, Dhall D, Cheng Z, et al. Murine model of Hirschsprungassociated enterocolitis II: surgical correction of aganglionosis does not eliminate enterocolitis. J Pediatr Surg 2010;45:206-211; discussion 211-212.

37. Volenec FJ, McCarthy RP, Humphrey M, Whitmore S, Humphrey LJ. Mouse colostomy model for studies on large bowel cancer. J Surg Oncol 1980;13:39-44. 